\title{
Les instructions du covid-19 et la nécessité de coopération des pays de l' Asie de l'Ouest en vue de sortie des crises
}

\author{
Mehdi Zakerian \\ Talieh Sakhamanesh
}

mzakerian@yahoo.com

Fondation internationale sur les dialogues et les études mondiales, FIDEM (France)

taliehsakhamanesh@hotmail.com

Islamic Azad University Science and Research Branch (Iran)

Recibido: 30/06/2021

Aceptado: $12 / 07 / 2021$

Résumé: La présente étude traite la question clé de savoir comment les pays de la région réagissent à une crise non politique de manière à ce qu'ils puissent résoudre, par la suite, les crises engendrées par d'autres problèmes au niveau national ainsi que dans la région de l'Asie de l'Ouest. Cet article vérifie l'hypothèse selon laquelle la coopération professionnelle dans le domaine de la prévention, du contrôle, de l'analyse, du traitement, de la vaccination et de l'échange de statistiques médicales est une nécessité urgente et non politique de la région qui peut ainsi favoriser le terrain pour l'extension, à l'avenir, de la coopération entre les pays d'Asie de l'Ouest dans d'autres secteurs. Cet article ayant une méthodologie descriptive-analytique, cherche à trouver des solutions pour plus de coopération et d'interaction face aux crises catastrophiques. En d'autres termes, cet article cherche à examiner les moyens de transformer la crise en une opportunité à saisir en Asie de l'Ouest.

Mots clés : Asie de l'Ouest, système régional, convergence, divergence, Covid-19

* Cómo citar este artículo: Zakerian, M. y Sakhamanesh, T. (2021). Les instructions du Covid-19 et la nécessité de coopération des pays de l'Asie de l'Ouest en vue de sortie des crises. Relaciones Internacionales, 30(60), 132.

https://doi.org/10.24215/23142766e132

Editor: Juan Alberto Rial, Instituto de Relaciones Internacionales Facultad de Ciencias Jurídicas y Sociales (Universidad Nacional de La Plata)
Entidad editora: Relaciones

Internacionales, es una publicación del Instituto de Relaciones Internacionales (Facultad de Ciencias Jurídicas y Sociales (Universidad Nacional de La Plata Argentina)

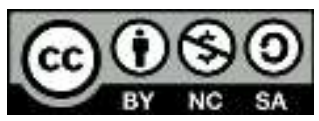

Reconocimiento-NoComercial Compartirlgual 4.0 Internacional (CC BY-NC-SA 4.0) 


\section{Las instrucciones del Covid-19 y la necesidad de cooperación de los países de Asia Occidental con miras a salir de las crisis}

\section{Mehdi Zakerian ${ }^{1}$, Talieh Sakhamanesh ${ }^{2}$}

Resumen: El presente estudio aborda la cuestión clave de comprender cómo los países de la región reaccionaron frente a una crisis no política de manera que puedan resolver, en adelante, las crisis engendradas por otros problemas, tanto en el orden nacional como en la región de Asia Occidental. Este artículo verifica la hipótesis según la cual la cooperación profesional en el campo de la prevención, el control, el análisis, el tratamiento, la vacunación y el intercambio de estadísticas médicas es una necesidad urgente y no política de la región, que puede así facilitar el terreno por ampliar, en el futuro, la cooperación en otros sectores entre los países de Asia Occidental. Este artículo, con una metodología descriptiva-analítica, busca encontrar soluciones para una mayor cooperación e interacción para enfrentar crisis catastróficas. En otras palabras, el presente artículo buscar examinar los medios para transformar la crisis en una oportunidad para ser aprovechar en Asia Occidental.

Palabras clave: Asia Occidental, sistema regional, convergencia, divergencia, Covid-19

\footnotetext{
${ }^{1}$ Professeur agrégée de droits de I'Homme à la faculté de droit et de sciences politiques de l'Université libre islamique(IAU), branche des sciences et des recherches à Téhéran (Iran). Ancien professeur invité au Faculté de droit à l'Université de Pennsylvanie; professeur invité à la faculté de droit à l'Université Paris II PanthéonAssas, membre associée du Centre d'études iraniennes, SOAS (Londres, États-Unis Royaume, depuis 2012 à nos jours) et également Président de la Fondation internationale sur les dialogues et les études mondiales, FIDEM. Actuellement il est redacteur- en - chef de I'International Studies Journal (ISJ) et du Journal of International Criminal Law (JICL).

${ }^{2}$ Doctorante en relations internationales, elle est professeur invitée du département des langues étrangères de l'universite libre islamique(AIU) branche Sciences et recherches à Téhéran. Elle est également responsable de la coopération économique et technologique internationale à I'AIU, Direction générale de l'économie basée sur la connaissance, Adjoint à la Recherche, à la Technologie et aux Innovations à Téhéran.
} 


\title{
Covid-19 instructions and Western Asian countries' need for cooperation to exit from the crisis
}

\section{Mehdi Zakerian, Talieh Sakhamanesh}

\begin{abstract}
This study tackles a key issue which involves understanding how the countries within the Western Asian region have reacted when faced by a non-political crisis so that they may in the future solve the crises created by other issues, whether they be at a national or at a regional level. This paper checks the hypothesis according to which professional cooperation in the field of prevention, control, analysis, treatment, vaccination and medical statistics exchange constitutes an urgent, non-political need in the region, which may pave the way to further cooperation in other sectors between Western Asian countries in the future. Using a descriptive and analytical methodology, this article seeks to find solutions for improved cooperation and interaction in order to deal with possible looming crises. In other words, it attempts to examine how to transform a crisis into an opportunity to be taken advantage of in Western Asia.
\end{abstract}

Key words: Western Asia, regional system, convergence, divergence, Covid-19 


\section{Introduction à l'énigme du coronavirus}

Le mode d'action d'un virus est au-delà des intentions politiques; les virus ne se propagent pas ou ne s'arrêtent pas en se soumettant aux intentions politiques. Réduire les affaires médicales et sanitaires aux affaires d'une nature politique est une lourde erreur. La communauté internationale ne doit pas désapprendre que lorsqu'il s'agit de confrontation aux maladies, surtout des maladies contagieuses dans un pays, cela ne doit pas être considéré comme le problème du pays en question, mais le problème de tous les pays. La déconsidération de cette question amènera l'ordre mondial des État-Nations à faire face à une crise. Que le chef d'un grand et puissant État comme les États Unis ne prend pas au sérieux la survenance d'une épidémie dans un autre coin du monde comme la Chine est un exemple de telles incuries. C'est ainsi que le coronavirus est d'abord devenu une crise et puis une énigme. Le coronavirus est un type de covid appelé covid-19. Ses dimensions ne sont pas entièrement connues. II entre dans divers organes du corps humain, rend le système immunitaire inopérant et, en atteignant les poumons, empêche l'oxygène de se circuler dans le cerveau, ce qui aboutit à la mort. Selon les chiffres de l'université Johns Hopkins, 41 millions de personnes ont contracté le virus dans le monde, dont plus d'un million sont décédées. II n'y a aucun pays au monde intouché par ce virus. Deux issues sont envisageables pour sortir de cette énigme : la première est la prévention. Cela veut dire qu'en suivant certaines consignes sanitaires, en respectant la distance sociale, en portant un masque, etc., l'on peut contrôler puis éliminer le virus. La deuxième, réside en fabrication du vaccin pour immuniser le corps. La première solution est suivie dans de nombreux pays. Bien qu'efficace pour casser la chaîne de transmission du virus, il n'a pas été en mesure de l'éliminer complètement, sauf dans un nombre limité des pays, comme la Chine. Dans le même temps, cette solution a abouti à l'accablement, aux confinements, à l'ennui et aux protestations des citoyens dans certains pays. La deuxième solution est la vaccination, qui a heureusement donné des résultats prometteurs grâce aux efforts de savants travaillant avec des sociétés transnationales. Les plus grandes sociétés pharmaceutiques transnationales du monde sont en train de faire des recherches dans ce domaine. Le Partenariat pour une action précoce tenant compte des risques (REAP) - un partenariat mondial qui vise à réunir les gouvernements, les bénéficiaires humanitaires, le développement et le climat afin de faire avancer l'agenda mondial sur la promotion d'une approche plus anticipative face aux catastrophes- a établi un groupe de travail sur covid-19 pour formuler des recommandations sur 1- I'anticipation des chocs durant la pandémie du covid-19; et 2- l'interaction avec les acteurs concernés afin de renforcer les mesures prévues dans le secteur de la santé. ${ }^{3}$ (Volet FAO du plan mondial de réponse humanitaire au covid-19, $2020: 32$ )

Ce qui est clair, c'est que de telles solutions mèneront à l'échec si elles ne sont pas accompagnées de la coopération des gouvernements. Le déplacement et les vaccins nécessitent tous les deux une interaction et une coopération sur le plan mondial. Les déplacements d'êtres humains d'un pays à l'autre, l'observance dans un pays et la non-

\footnotetext{
${ }^{3}$ Traitement des effets du covid-19 sur les crises alimentaires-avril-décembre 2020)
} 
observance dans un autre, peuvent entraver un contrôle du virus via le respect de la distance sociale. En outre, les pays ont besoin de plus de coopération dans les recherches et les études médicales.

Tableau 1. Types d'outils des politiques au temps de pandémie de Covid-19 (Brian Y. An et Shui-Yan Tang, 2020 : 796) (Leçons tirées des réponses au COVID-19 dans l'Asie de l'Ouest: infrastructures institutionnelles et outils d'élaboration des politiques durables)

\begin{tabular}{|c|c|}
\hline $\begin{array}{l}\text { Types d'outils d'élaboration des } \\
\text { politiques }\end{array}$ & Options sélectionnées \\
\hline \multirow[t]{5}{*}{ Essai } & $\begin{array}{l}\text { Géographie : compréhensive vs axée sur un groupe (c.-à- } \\
\text { d. concentrée sur une zone) }\end{array}$ \\
\hline & $\begin{array}{l}\text { Couverture : toute personne présentant des symptômes } \\
\text { suspects par rapport à des personnes données pré- } \\
\text { sentant des } \\
\text { symptômes respiratoires }\end{array}$ \\
\hline & $\begin{array}{l}\text { Admissibilité : citoyens vs non-citoyens (c.-à-d. immi- } \\
\text { grants sans citoyenneté); non-criminels vs. } \\
\text { criminels }\end{array}$ \\
\hline & Coût : gratuit (universel) vs partage des coûts \\
\hline & $\begin{array}{l}\text { Accessibilité : centres désignés ou sites de conduite/de } \\
\text { passage par rapport à l'approbation des médecins après } \\
\text { consultation }\end{array}$ \\
\hline \multirow[t]{3}{*}{$\begin{array}{l}\text { Restrictions de déplacement et } \\
\text { des transports }\end{array}$} & $\begin{array}{l}\text { Degré de restrictions : ordonnance de séjour à domicile } \\
\text { obligatoire vs ordonnance de séjour à domicile volontai- } \\
\text { re vs non restriction }\end{array}$ \\
\hline & Géographie : confinement national vs confinement local \\
\hline & Lieux : domicile, lieu de travail, transports en commun \\
\hline Contrôle des frontières & $\begin{array}{l}\text { Cible d'interdiction d'entrée : tous les pays vs certains } \\
\text { pays (régions) dans les groupes à haut risque vs aucun } \\
\text { contrôle aux frontières }\end{array}$ \\
\hline Quarantaine (et recherche des & Surveillance : surveillée vs volontaire \\
\hline
\end{tabular}




\begin{tabular}{|c|c|}
\hline \multirow[t]{3}{*}{ contacts) } & Lieux : établissements publics vs hôpitaux vs domiciles \\
\hline & $\begin{array}{l}\text { Coûts : paiement par la personne en quarantaine vs aide } \\
\text { gouvernementale }\end{array}$ \\
\hline & $\begin{array}{l}\text { Méthodes : entretiens et visites en personne vs techno- } \\
\text { logie basée sur une application (GPS) vs bracelets élec- } \\
\text { troniques }\end{array}$ \\
\hline $\begin{array}{l}\text { Groupes prioritaires pour le } \\
\text { traitement }\end{array}$ & $\begin{array}{l}\text { Groupes : citoyen vs non-citoyen ; titulaire d'assurance } \\
\text { vs non titulaire; personnes âgées vs non âgées }\end{array}$ \\
\hline $\begin{array}{l}\text { Distanciation sociale et autres } \\
\text { mesures d'hygiène (par exem- } \\
\text { ple, le port du masque) }\end{array}$ & $\begin{array}{l}\text { Application : obligatoire (par la loi) vs laissée aux entre- } \\
\text { prises privées vs pratiques volontaires }\end{array}$ \\
\hline \multirow[t]{2}{*}{$\begin{array}{l}\text { Campagne d'information publi- } \\
\text { que }\end{array}$} & $\begin{array}{l}\text { Sources : messages textes mobiles ; journaux; panneaux } \\
\text { d'affichage; publicités télévisées; médias sociaux; page } \\
\text { d'accueil du gouvernement }\end{array}$ \\
\hline & Matériel : dessins animés; mots; vidéos \\
\hline Entreprise (et école) & $\begin{array}{l}\text { Exploitation : entreprises ouvertes comme d'habitude vs } \\
\text { industries essentielles autorisées à ouvrir uniquement } \\
\text { (c'est-à-dire, non essentielles fermées) vs toutes fer- } \\
\text { mées ; école ouverte vs fermeture temporaire }\end{array}$ \\
\hline $\begin{array}{l}\text { Restrictions pour les rassem- } \\
\text { blements de masse }\end{array}$ & $\begin{array}{l}\text { Seuil : numéro universel appliqué à l'ensemble du pays } \\
\text { vs restriction basée sur la densité } \\
\text { Type : tous les rassemblements publics interdits vs les } \\
\text { rassemblements privés également interdits }\end{array}$ \\
\hline
\end{tabular}

La vie en Asie occidentale a toujours été parsemée de souffrances et de difficultés. Les habitants de cette région ont chacun vécu au moins une crise régionale durant leur vie. C'est pourquoi sortir de la crise devient parfois un rêve. Quelle est la solution si l'on considère cette hypothèse comme un désavantage pour la région du Moyen-Orient? Comment sortir de la crise en Asie occidentale? Comment la coopération est-elle possible dans cette région ? Quelles opportunités et menaces, le coronavirus a créé en Asie occidentale en tant qu'une crise mondiale? Quelles décisions et mesures sont nécessaires pour endiguer le covid-19? En tant qu'un fait non politique, la maladie à coronavirus exige la coopération des régimes politiques de la région. Certaines de ces questions sont des questions secondaires d'une question clé dans cette étude : Comment les pays d'Asie occidentale réagissent-ils à une crise non politique (coronavirus) de façon à ce qu'ils puissent résoudre des crises issues d'autres problèmes au niveau national et à ce de la région d'Asie occidentale? 
En réponse à cette question clé, l'auteur vérifie l'hypothèse selon laquelle la coopération professionnelle dans le domaine de la prévention, du contrôle, de l'analyse, du traitement, de la vaccination et de l'échange des statistiques médicales en tant qu'une nécessité urgente non politique de la région peut rendre le chemin propice aux pays d'Asie occidentale pour coopérer dans d'autres secteurs.

Les théories qui régissent des relations et des conflits dans les relations internationales montrent que l'augmentation de la coopération et celle de la tension dans toute région dépendent des politiques qui sont mises en œuvres. Jean Monnet, diplomate et professeur d'université français a réfléchi en 1948 et 1949 à une issue pour mettre fin au conflit franco-allemand aboutissant aux ravages de la Seconde Guerre mondiale. II a réfléchi à un fait technique : le charbon et l'acier. Cette question sera développée et détaillée dans les prochaines parties de cette étude. Mais en peu de mots, Monnet croit que des résultats politiques peuvent être tirés d'un fait non politique. Par conséquent, l'hypothèse élaborée dans cette étude utilise la question de coronavirus en tant qu'un phénomène non politique dans la communauté internationale pour arriver à une solution aux crises politiques dans la région de I'Asie occidentale. La méthodologie de cette recherche est descriptiveanalytique mais l'utilisation des outils statistiques et des rapports de I'Organisation mondiale de la santé sur le taux de mortalité amène cet article à dépasser la méthodologie descriptive-analytique et à s'inscrire dans le champ des recherches par sondage. Cependant l'examen de cette hypothèse repose sur une méthode descriptive-analytique. Les outils de cette recherche et la méthode de collecte des données sont des sources bibliographiques et des chiffres fiables qui servent à enrichir l'analyse des sujets. L'objectif de cette étude est de trouver des solutions pour plus de coopération face aux crises catastrophiques. En d'autres termes, les auteurs cherchent des solutions pour transformer la crise en opportunité en Asie occidentale. Cet article met l'accent sur l'hypothèse selon laquelle la coopération dans les affaires non politiques tels que le contrôle du coronavirus, son traitement et sa prévention pourrait ouvrir la voie à une future coopération politique.

\section{Conséquences géopolitiques de la pandémie du Covid-19}

\section{i. Diplomatie numérique}

La diplomatie au début du $21^{\mathrm{e}}$ siècle était déjà très différente de celle des années précédentes. Un nouveau style de langage, ainsi que l'utilisation des médias sociaux et l'augmentation de la désinformation avaient créé un environnement diplomatique qui semblait plus conflictuel, plus antagoniste et moins axé sur ce pour lequel elle avait été créé : la communication, l'échange de vues et le développement des solutions communes parmi les États.

En apparence, covid-19 a mis fin à la diplomatie telle qu'elle était connue : la forte réduction de déplacements, ainsi que les restrictions aux contacts sociaux ont fait que les réunions, qui sont la pierre angulaire de la pratique diplomatique, n'étaient plus possibles, ou seulement à un niveau très limité. Début mars, I'Assemblée générale des Nations Unies, le sommet de I'ASEAN, la Conférence des Nations Unies sur le changement climatique, le sommet UE-Chine, le sommet UE-Inde, le Congrès du Conseil de l'Europe et une multitude 
de réunions du Conseil européen ont été reportés ou annulés dans les premiers mois de la crise. Aux Nations Unies, les réunions du Conseil de sécurité ont été annulées ainsi qu'une session du Conseil des droits de l'homme et une série des réunions de groupes de travail. (Département thématique des relations extérieures, Direction générale des politiques externes de l'Union, $2020: 36$ )

Une autre point clé est la possibilité d'effectuer une recherche des contacts transfrontaliers et régionaux. De nombreuses économies utilisent désormais des outils numériques de recherche des contacts. II existe cependant un large éventail d'outils adoptés, des systèmes centralisés aux systèmes décentralisés et l'utilisation de différentes technologies telles que les codes QR ou Bluetooth. Les systèmes variables d'une économie à l'autre rendent difficile l'utilisation d'applications de recherche de contacts pour les déplacements transfrontaliers. Par conséquent, la promotion du tourisme est et sera en premier lieu une tâche commune, et elle nécessite plus que jamais une coopération régionale. (Matthias Helble et Ana Fink, 2020 : 12) (Reprise du tourisme pendant la pandémie de covid-19)

\section{ii. L'activisme démocratique à venir}

L'activisme mondial n'a pas été épargné par la pandémie: alors qu'en 2019 - étiqueté "l'année de la manifestation de rue » - le plus petit nombre d'événements de protestation par semaine dans toutes les régions sûres de l'Amérique du Nord, de l'Europe et de l'Australie dépassait le millier, en mars et à la mi-avril, ce nombre est tombé à environ la moitié de ce chiffre. (Département thématique des relations extérieures, Direction générale des politiques externes de l'Union, 2020 : 42) (Conséquences géopolitiques de la pandémie de Covid-19)

\section{iii. Incertitudes principales}

-Si l'activisme politique augmente, quels sont ses impacts sur les systèmes politiques et l'instabilité? L'activisme peut avoir des effets fortement déstabilisants sur les sociétés et les économies, mais ces effets peuvent aussi être des éléments importants de changement et même de réforme. Là où l'activisme est constructif et non violent, ses effets sont plus susceptibles de conduire à des changements profonds. (Département thématique des relations extérieures, Direction générale des politiques externes de l'Union, 2020 : 44) (Conséquences géopolitiques de la pandémie de Covid-19)

-La pandémie peut-elle stimuler une nouvelle façon d'aborder la démocratie ? S'il est devenu courant de décrire les démocraties comme des systèmes en crise, ce n'est pas le système lui-même qui est remis en cause mais la manière dont il est pratiqué. Les troubles provoqués par la pandémie pourraient très bien conduire à d'importantes réformes à cet égard. (Conséquences géopolitiques de la pandémie de Covid-19)

-Les restrictions des principes démocratiques sont-elles irréversibles ? Le recul démocratique constaté avant et pendant la pandémie pourrait être le signe d'une tendance à plus long terme, voire le retour aux circonstances du début des années 2000. D'autre part, la tendance pourrait s'inverser dans les années à venir grâce à l'amélioration des niveaux de participation, à la réduction des inégalités et à une gouvernance plus réactive. (Con- 
séquences géopolitiques de la pandémie de Covid-19)

\section{iv. Analyse de l'avenir de l'ordre régional de l'Asie occidentale étant donnée les impacts de la pandémie du coronavirus}

La pandémie du coronavirus et ses impacts font partie des évolutions qui peuvent changer l'avenir de l'ordre international et par conséquent, l'ordre régional d'Asie occidentale, et ont créé différentes visions, dont chacune reflète une image de l'avenir de cet ordre. Cet article a pour objectif l'analyse et la présentation d'une image de l'avenir de l'ordre régional d'Asie occidentale en considérant les impacts de la pandémie du coronavirus et la mise en place d'une solution à ce problème. L'ordre régional est constitué d'une totalité cohérente ou incohérente d'États homogènes qui ont des caractéristiques et des éléments structurels relativement visibles ou invisibles et qui nouent des relations bilatérales ou plurilatérales avec des totalités inférieures (pays), supérieures (ordre international) et homologues (ordres régionaux). L'ordre régional comporte des parties à la fois séparées et connexes, lesquelles consistent en une partie centrale (acteurs principaux et secondaires) et une partie marginale et périphérique (petits acteurs et éléments intervenants transrégionaux).

Vu la pandémie du coronavirus qui sévit aux quatre coins du monde y compris en Asie occidentale, il existe deux visions et images opposées pour l'analyse de l'avenir de l'ordre régionale de I'Asie occidentale. Ceux qui se rangent du côté de la première vision estime que l'ordre régional de l'Asie occidentale après la fin de la pandémie du coronavirus sera orienté vers réduction du rôle des gouvernements, extension du rôle des institutions régionales, développement du processus du régionalisme en tant qu'un élément de mondialisation, remplacement du principe d'auto-assistance et d'unilatéralisme par le multilatéralisme, changement des modèles de communication soit le passage de la divergence à la convergence et que finalement les politiques sécuritaires et les rivalités géopolitiques perdront de l'importance en raison des intérêts mutuels et de l'interdépendance économique. Mais selon l'image décrite par la deuxième vision de l'avenir de l'ordre régional d'Asie occidentale, les impacts de la pandémie de coronavirus pousseront l'ordre régional à se diriger de plus en plus vers l'instabilité régionale et la divergence en raison des crises économiques, sanitaires et sociales déclenchées par de diverses évolutions. De ce fait, l'on constatera également l'expansion du terrorisme et l'intensification des rivalités géopolitiques mais surtout la présence et l'intervention des puissances étrangères dans cet ordre régional. Compte tenu des éléments mentionnés, l'on constatera de plus en plus le rôle du gouvernement en tant qu'acteur régional principal et dominant, un modèle de communication de nature de rivalité et de divergence, la priorité du régionalisme sur la mondialisation et la primauté de la politique de sécurité sur les politiques économiques et un mouvement vers l'auto-assistance. La deuxième vision tout en s'appuyant sur l'approche réaliste analyse d'une certaine façon, l'avenir de l'ordre régional en Asie occidentale.

Donc dans ce genre de vision, les réalistes croient que le monde verra la renaissance et le retour de l'État-nation et le renforcement de son Pouvoir, et que la mondialisation libérale basée sur l'interdépendance sera affaiblie. Malgré la nécessité d'une coopération internationale, les gouvernements n'y parviendront pas en raison de la logique de l'arro- 
gance et de l'indépendance. Les réalistes critiquent depuis longtemps la mondialisation libérale, arguant que l'interdépendance ne peut pas changer la nature anarchique de l'ordre et de la politique internationale; Car autant elle présente des avantages, autant elle augmente la vulnérabilité des pays. Ainsi, un théoricien réaliste comme Stephen Walt pensent que la mondialisation libérale doit être reconstruite sur la base de l'ordre libéral international et de l'ordre domestique, et que nous verrons le retour de l'État-nation; Et cela de manière à ce que le village planétaire forgé par la mondialisation s'affaiblisse et même prenne fin. L'élection de Donald Trump dans les présidentielles précédentes aux États-Unis ou la question du Brexit dans I'Union européenne étaient en soi le signe d'un retour au nationalisme et au mercantilisme. La crise du coronavirus renforcera également ce courant.

\section{Impacts de la pandémie de coronavirus sur les pays d'Asie occidentale}

\section{i. Fragilisation du talon d'Achille des États faibles}

Les pays d'Asie occidentale ont subi les conséquences de la pandémie au niveaux différents, étant donné les ressources naturelles telles que le pétrole et le gaz, la taille de la population, la situation du tourisme, la sécurité de l'emploi, la capacité économique et sanitaire, la stabilité politique, le développement politique et économique et le bien-être public. Ainsi, selon les statistiques mondiales, les conséquences les plus importantes de la pandémie de coronavirus sont le chômage d'un grand nombre de personnes, la faillite d'entreprises privées et publiques, l'endommagement des économies nationales, l'augmentation de la pauvreté, l'affaiblissement de la sécurité humaine et le mécontentement des groupes sociaux à l'égard du gouvernement central. Parmi les pays d'Asie occidentale, il y a des États intitulés fragiles ou en faillite, que Cohen juge d'instables en raison d'un développement défavorable, d'un taux de chômage élevé, des tensions ethniques et de la faiblesse de l'autorité centrale. Ils se dirigent vers une crise interne, l'insécurité et le chaos, qui peuvent également menacer la sécurité régionale et la sécurité internationale, ce qui aboutira à son tour à l'intervention des puissances étrangères dans ces pays. D'une manière générale, nous pouvons prédire que des gouvernements tels que I'Irak et la Syrie, qui sont considérés comme des États en faillite, en raison des crises mentionnées ci-dessus, avec la propagation du coronavirus et ses impacts sur les secteurs économique, sanitaire et politique de ces pays auront à l'avenir, un ordre régional de plus en plus fragile et en faillite. Et cela parce que ces pays ne disposent pas des infrastructures appropriées pour faire face à ce type de crise et ne bénéficient pas d'un soutien économique élevé par rapport aux pays du golfe Persique comme l'Arabie Saoudite et les Emirats Arabes Unis, et donc les conséquences négatives de la pandémie auront un impact plus important sur ces pays.

\section{ii. Expansion du terrorisme}

Étant que la propagation du terrorisme, surtout celle de l'Etat islamique en Irak et en Syrie, était due à la fragilité de ces deux gouvernements, ainsi qu'à l'insatisfaction et l'écart entre les classes sociales et le pouvoir central, l'on peut attendre à ce que les effets 
négatifs et catastrophiques de coronavirus en Irak et en Syrie et dans les pays présentant cette caractéristique, aplanisse le chemin pour la propagation du terrorisme sur le territoire de ces pays, A titre d'exemple, l'on peut indiquer les attentats suicides de deux terroristes sur le bazar de Baghdâd durant la crise de coronavirus. L'État islamique a également subi plusieurs défaites avant la pandémie de coronavirus, mais pendant ces derniers mois, il a essayé d'organiser ses troupes, de reprendre ses actes terroristes en cherchant des occasions et même d'étendre ses actes dans d'autre pays en crise interne comme le Yémen. Avec le soutien des pays de la région et autres pays tels que l'Arabie saoudite et les États-Unis, ce groupe terroriste s'évertue souvent à poursuivre ses actes en cherchant des instabilités internes et des changements dans les priorités politico-sécuritaires des gouvernements. Par conséquent, si les États fragiles n'arrivent pas à établir une certaine stabilité à l'intérieur ainsi qu'à leurs frontières en raison de faiblesses politiques, économiques et sécuritaires dues aux impacts de la pandémie du coronavirus, dans un avenir proche, l'ordre régional d'Asie occidentale se verra davantage le champ de l'expansion du terrorisme et des attentats meurtriers que ces dernières années.

\section{iii. Renforcement des rivalités géopolitiques}

Alors que des pays comme l'Irak, la Syrie et le Yémen se fragilisent de plus en plus en raison des effets négatifs de la pandémie du coronavirus, l'on s'attend à ce que les acteurs centraux dans l'ordre de I'Asie occidentale, à savoir la République islamique d'Iran, l'Arabie saoudite et même la Turquie s'engagent aux rivalités géopolitiques plus sérieuses. De son côté, I'Iran empêche la fragilisation des États susmentionnés et poursuit cette stratégie afin d'étendre son influence régionale et de renforcer sa position régionale. L'Arabie saoudite, pour sa part, poursuit également ses propres intérêts, essayant d'empêcher l'influence régionales iraniennes en acculant lesdits gouvernements en faillite afin de modifier finalement le rapport de force en sa faveur et celle de ses alliés. Au sujet de la propagation du terrorisme, il convient d'ajouter que l'Iran essaie de soutenir ses partenaires stratégiques en fournissant des soutiens économiques, politiques et militaires, tandis que l'Arabie saoudite, en coopération avec les États-Unis, essaie de remporter cette rivalité géopolitique en imposant des sanctions telles que César et d'autres sanctions économiques et politiques contre la Syrie, en lançant des attaques militaires contre le Yémen, ainsi qu'en apportant d'énormes soutiens économiques et militaires aux groupes terroristes. Les pays fragiles comme l'Irak, la Syrie et le Yémen ont une situation géopolitique très importante et il convient donc de prêter attention à l'importance de leurs frontières géopolitiques et de leur fragilité à l'avenir. Un autre point qu'il faut souligner est les efforts de l'Arabie saoudite et de ses alliés régionaux pour la déstabilisation et la prolongation de la crise dans les États fragiles afin qu'ils puissent tirer le meilleur parti du changement de pouvoir dans ces pays, mais l'Iran, avec le ferme soutien qu'il apporte aux gouvernements légitimes de ces régions essaiera d'établir une certaine stabilité relative dans ces pays. Ainsi, l'on peut pronostiquer que dans la période post-covid, l'ordre régional verra des tensions grandissantes entre les rivaux géopolitiques.

\section{iv. Expansionnisme des pouvoirs transrégionaux}

Comme nous venons d'indiquer, Cohen croit que les gouvernements en faillite, parce 
que leurs crises peuvent s'étendre aux niveaux régional et même international, obligent les pouvoirs transrégionaux à intervenir en vue de les diriger. Dans l'étude de l'ordre régional de l'Asie occidentale, l'un des facteurs toujours présents est l'intervention des puissances transrégionales dans cette région. Il s'avère nécessaire de souligner qu'en raison de leur emplacement stratégique, de leurs ressources naturelles abondantes et de leurs crises sécuritaires, ces régions ont fait l'objet de l'intervention de ces puissances autant que possible, de sorte que leur intervention et leur influence croissantes semblent faire partie intégrante de l'ordre de l'Asie occidentale. Les puissances interventionnistes telles que les États-Unis et la Russie tentent de renforcer et de poursuivre leurs intérêts nationaux et leur influence transrégionale en intervenant dans la gestion de la crise causée par la pandémie du coronavirus, étant donné les impacts de la pandémie du coronavirus sur les pays en faillite d'Asie occidentale, le déclenchement des crises économiques, sociales, sanitaires et sécuritaires prévisibles et la possibilité d'un changement dans l'équilibre des puissances parmi les États centraux de cet ordre régional. II faut rappeler que l'intervention de ces pays minore de plus en plus, les composantes de convergence, de coopération et de politiques multilatérales et pousse l'ordre régional à devenir de plus en plus dépendant à l'égard de l'ordre international. Ainsi, l'intervention de ces pouvoirs les amène à poursuivre des politiques convergentes avec un pouvoir régional, et cela conduira à l'établissement d'un modèle de communication plus divergente et à un type d'activisme centrée sur les gouvernements. Car un ordre régional anarchique rend les gouvernements peu optimistes quant aux unions et institutions régionales, et le processus de mondialisation dans cet ordre régional est éclipsé par des questions de sécurité. Donc, à l'inverse des chercheurs et des politiciens qui pensent que la pandémie de coronavirus fait passer les gouvernements de la divergence à la coopération et à l'interdépendance, et que dans un ordre régional, les intérêts communs poussent leurs politiques à devenir plus favorables, évidemment la pandémie du coronavirus, a pour conséquence la non coopération et la rivalité surtout parmi les pays fragiles.

\section{Résultats de l'étude : coopérations régionales en tant qu'une issue de crise}

La pandémie de coronavirus et ses impacts sont sans aucun doute un événement majeur aux dimensions sanitaire-médicale, socio-économique et politico-sécuritaire et ont également affecté l'ordre mondial et régional. L'une de ces régions, qui a subi de diverses crises avant la pandémie de coronavirus et dont les crises ont doublé avec cette pandémie, est l'ordre régional d'Asie occidentale. Dans l'ordre régional de l'Asie occidentale, cette pandémie a fait souligné l'importance de la place des États en tant qu'acteurs dominants. Les effets du coronavirus dans différents pays peuvent être étudiés sous différents aspects et degrés. Au cours de ces dernières années, de diverses évolutions intervenues dans l'ordre international ont également modifié les ordres régionaux. Des événements tel que la fin de la guerre froide et les attentats du 11 septembre ont fait changer les régimes régionaux, surtout ceux de l'Asie occidentale. La pandémie de coronavirus est un exemple de ces importantes évolutions mondiales qui aura un impact aux différents niveaux nationaux, régionaux et internationaux. Avec ces explications, cet article a pour l'objectif de répondre à la question de savoir quels effets a eu la pandémie virale sur l'ordre régional de l'Asie occidentale, ou en d'autres termes, étant donné les impacts de la pandémie du co- 
ronavirus, quel sera l'avenir de l'ordre régional de l'Asie occidentale. Dans cet article, nous cherchons à brosser un tableau de l'avenir de l'ordre régional d'Asie occidentale en considérant les impacts de la pandémie de coronavirus et à mettre en avant une issue à ce problème.

\section{Confinement}

Le confinement est l'un des moyens pour faire face immédiatement à coronavirus: Restriction de circulation, fermeture des frontières, isolement, mesures de salubrité publique et respect de l'hygiène. Bien que cette solution conduise de manière générale au mécanisme de l'isolement, mais c'est une solution efficace pour endiguer la propagation du covid-19. Dans ce cadre, un effort pour le maintien de la santé des citoyens nécessite une réglementation stricte.

Tableau 2. Proportion d'écoles, par région et niveau d'enseignement, ayant accès aux infrastructures de base et accès aux nouvelles technologies à des fins éducatives. (Luis Lázaro, 2020: 6) (Le droit à l'éducation et aux Technologies de l'information (ICT) et de la communication pendant le covid-19 : Une perspective internationale)

\begin{tabular}{|l|c|c|c|c|c|c|}
\hline \multirow{2}{*}{$\begin{array}{l}\text { Groupes ré- } \\
\text { gionaux }\end{array}$} & \multicolumn{2}{|c|}{ Électricité } & \multicolumn{2}{c|}{ Ordinateurs } & \multicolumn{2}{c|}{ Internet } \\
\cline { 2 - 7 } & Primaire & Secondaire & Primaire & Secondaire & Primaire & Secondaire \\
\hline Océanie & $45.89 \%$ & $98.16 \%$ & $65.81 \%$ & $84.74 \%$ & $68.32 \%$ & $85.38 \%$ \\
\hline Asie centrale & $99.97 \%$ & $99.26 \%$ & $95.71 \%$ & $95.01 \%$ & $78.35 \%$ & $78.76 \%$ \\
\hline Asie orientale & $97.16 \%$ & $97.92 \%$ & $91.72 \%$ & $97.09 \%$ & $91.30 \%$ & $94.43 \%$ \\
\hline Asie du sud est & $83.82 \%$ & $93.22 \%$ & $48.03 \%$ & $66.51 \%$ & - & $60.06 \%$ \\
\hline Asie du sud & $52.22 \%$ & $67.60 \%$ & $15.95 \%$ & $41.56 \%$ & - & $46.63 \%$ \\
\hline $\begin{array}{l}\text { Asie occidenta- } \\
\text { le }\end{array}$ & $91.10 \%$ & $94.61 \%$ & $80.77 \%$ & $91.79 \%$ & $79.64 \%$ & $86.40 \%$ \\
\hline $\begin{array}{l}\text { Europe et } \\
\text { Amérique du } \\
\text { Nord }\end{array}$ & $85.70 \%$ & $79.60 \%$ & $63.22 \%$ & $59.86 \%$ & $66.82 \%$ & $73.54 \%$ \\
\hline $\begin{array}{l}\text { Afrique du } \\
\text { Nord }\end{array}$ & - & $99.89 \%$ & - & $98.67 \%$ & - & $96.35 \%$ \\
\hline
\end{tabular}




\begin{tabular}{|l|c|c|c|c|c|c|}
\hline $\begin{array}{l}\text { Afrique } \\
\text { subsaharienne }\end{array}$ & $32.47 \%$ & $47.20 \%$ & - & - & - & - \\
\hline $\begin{array}{l}\text { Amérique } \\
\text { latine et } \\
\text { Caraïbes }\end{array}$ & $87.91 \%$ & $92.14 \%$ & $60.63 \%$ & $73.61 \%$ & $43.36 \%$ & 63.49 \\
\hline
\end{tabular}

Source : Extrait de la base de données des Objectifs de développement durable (SDG) des Nations Unies (2020).

Ainsi, l'on peut conclure que les politiques orientées vers l'équité et l'éducation inclusive sont désormais plus que jamais nécessaire pour garantir et renforcer le droit à l'éducation dans le cadre de la réalisation des objectifs de développement durable des Nations Unies dans les régions d'Asie occidentale. (Luis Lázaro, 2020: 13)

\section{Une coopération internationale pour résoudre les problèmes et les défis du $21^{\mathrm{e}}$ siècle}

La pandémie de covid-19 a menacé non seulement notre santé et nos systèmes de santé, mais aussi l'économie mondiale, la protection sociale, les droits de l'homme, la stabilité et les perspectives de développement durable de milliards de personnes. Elle met en évidence la profondeur de notre interconnexion et les lacunes dans notre capacité à fournir des biens publics mondiaux essentiels, tels que la santé publique, le développement durable, un environnement propre et une planète saine, une économie mondiale qui fonctionne et la paix pour tous. D'un autre côté, là où il y a la détermination et la vision, nous avons la capacité de nous rassembler en tant que communauté mondiale pour forger des solutions collectives à des problèmes communs. Les objectifs de développement durable et l'Accord de Paris étaient des expressions claires de la volonté mondiale de créer un avenir meilleur, plus égal, plus inclusif et plus durable. Mais il faudra un effort concerté pour construire le monde envisagé par ces accords, d'autant plus que nous sortons de cette pandémie. Alors que nous traçons la voie de la sortie de la crise du covid-19 et un avenir meilleur, nous devons également renforcer et renouveler les structures de coopération au niveau mondial afin de mieux nous préparer à la prochaine crise de ce type.

\section{Consensus: les approches de droits de l'homme garantissent de meilleurs résultats pour sortir du problème du Coronavirus en Asie occidentale}

\section{i. Les régions de l'Asie occidentale}

La pandémie de covid-19 est une crise humaine qui devient rapidement une crise des droits de l'homme. En tant que tels, les droits de l'homme doivent diriger la réponse et le rétablissement de covid-19. Une perspective des droits de l'homme peut aider à vaincre la pandémie, en mettant l'accent sur ceux qui risquent d'être laissés pour compte. Premièrement, la révélation des faiblesses caractéristiques dans la fourniture de services publics 
et les inégalités structurelles qui en empêchent l'accès, et le soulignement de cet point important qui est la manière dont les gouvernements peuvent s'assurer de meilleurs résultats en prenant en compte les droits de l'homme dans leurs réponses. Deuxièmement, la responsabilité des États ne devrait pas être discriminatoire dans le traitement et l'application des protocoles; Nous devons prêter attention aux personnes les plus vulnérables et marginalisées, car si le virus persiste dans un pays ou une société, il restera une menace pour nous tous. Troisièmement, un engagement efficace exige que les gens soient conscients et impliqués dans les décisions qui les concernent. La réalisation de cet objectif dépend de la transparence. Quatrièmement, les mesures d'urgence devraient être nécessaires, raisonnables, limitées dans le temps et proportionnées, et être considérées comme telles. La meilleure réponse est celle qui répond proportionnellement aux menaces immédiates tout en protégeant les droits de l'homme et la primauté du droit. La pandémie ne doit pas être utilisée comme prétexte pour des restrictions sur l'espace civique ou d'autres mesures non justifiées par le virus lui-même. Cinquièmement, l'unité et la solidarité internationales sont essentielles pour une réponse efficace. Sixièmement et enfin, en respectant les droits de l'homme en cette période de crise, nous construirons des solutions plus efficaces et inclusives pour l'urgence d'aujourd'hui et le rétablissement du demain, conformément à l'Appel à l'action sur les droits de l'homme. Au niveau des pays, les Nations Unies, dirigées par le Haut-Commissariat aux droits de l'homme (OHCHR), s'efforcent d'inscrire les droits de l'homme au cœur de la réponse des États, des partenaires des Nations Unies, de la société civile et du secteur privé, et veiller à ce que les impacts du covid19 sur les droits de l'hommes soient traités efficacement pendant la phase de rétablissement, avec des mesures ciblées pour soutenir les personnes marginalisées et les plus vulnérables. Cela comprend, par exemple, le suivi des problèmes spécifiques des droits de I'homme qui prennent une urgence accrue dans le contexte de covid-19, comme les conditions de surpeuplement dans les prisons. Le OHCHR a fourni des conseils ciblés à l'intention des États et d'autres partenaires pour orienter les réponses et atténuer ces défis en matière de droits de l'homme.

\section{ii. Institutions mondiales}

Les pays peuvent avancer principalement sur la base de l'épidémiologie ou sur l'épidémiologie en combinaison avec d'autres considérations; cependant, un plan clair et transparent décrivant les facteurs pris en compte est essentiel. Idéalement, ces plans devraient indiquer explicitement les niveaux ou les phases d'assouplissement des restrictions, les critères de passage au niveau ou à la phase suivant, et les mesures de confinement que chaque niveau ou phase implique. Chaque pays doit disposer d'un système efficace de recherche, de test, de traçage, d'isolement et de prise en charge. Au fur et à mesure que de nouvelles preuves seront disponibles, certaines de ces stratégies pourraient aider les pays à maintenir la suppression virale et à éviter un retour à un confinement complet. (Melisa Mei Jin Tan et al., 2020 :1532) Vu la nature en évolution rapide de la pandémie et des mesures prises pour y répondre, dans un esprit de coopération internationale, il faut fournir des préceptes dans les politiques sanitaires qui permettent d'apprendre des conditions et des stratégies des pays de la région, et cela dans le but de facilitation d'apprentissage et de l'orientation des politiques postérieures sur la mission 
complexe et problématique de levée de confinement de covid-19. La réduction des confinements est un enjeu qui doit être dirigé avec une vigilance constante. L'on espère que les pays continueront à partager leurs expériences, leurs informations et leurs stratégies alors qu'ils répondent à ce virus qui ne connaît pas de frontières.

\section{iii. Reprise du tourisme durant la pandémie de coronavirus}

Pour la reprise du tourisme, les gouvernements de la région de l'Asie occidentale, doivent développer une approche progressive. Plus les gouvernements réussissent à contenir le virus, plus il est probable qu'ils engagent des négociations pour ouvrir des voyages avec des partenaires qui ont également réussi. Ces négociations pourraient aboutir à la mise en place de corridors verts permettant les déplacements entre les pays partenaires suivant des protocoles sanitaires stricts. Ces accords bilatéraux peuvent ensuite être progressivement étendus à des accords plurilatéraux. Dans ce qui suit, nous proposons une analyse de cette approche par étapes. Tout d'abord, nous analysons le potentiel du tourisme intérieur, puis des accords de voyage bilatéraux et enfin des potentiels de voyage sous-régionaux.

\section{a) Scénario 1 : Promouvoir le tourisme intérieur}

Compte tenu des restrictions de voyage internationales et de la peur de l'infection, le tourisme intérieur offre de meilleures chances de rebond au début de la reprise. De nombreux gouvernements cherchent des moyens de stimuler le tourisme intérieur afin de soutenir l'industrie touristique en difficulté et d'améliorer la situation économique et cela est une stratégie appropriée. (Matthias Helble et Anna Fink, 2020 :5)

\section{b) Scénario 2 : Négocier des bulles de voyage avec des partenaires privilégiés}

Une autre option pour que les pays relançant le tourisme consiste à établir des bulles de voyage ou des corridors verts avec d'autres pays. Les bulles de voyage, dans notre analyse, sont des accords par les signataires pour ouvrir leurs frontières aux visiteurs d'une économie ou d'économies partenaires. Les bulles de voyage peuvent être réservées aux voyages d'affaires ou inclure également les voyages d'agrément. Elles précisent souvent des dispositions sur les protocoles sanitaires à respecter lors de la sortie et de l'entrée sur le territoire. L'accès peut donc être réciproque ou unilatéral. Elles peuvent être formés entre deux ou plusieurs partenaires. (Matthias Helble et Anna Fink, 2020 :7)

\section{c) Scénario 3 : Établir des bulles de voyage sous-régionales}

Des bulles de voyage sous-régionales ou multilatérales sont également en cours de discussion et peuvent être considérées comme une prochaine étape vers de multiples accords bilatéraux. Les communautés régionales d'Asie et d'Océanie ont une histoire de coopération en matière de tourisme et de facilitation des voyages, et parmi eux, nombreux sont ceux qui négocient pour aider à répondre à la crise créée par covid-19. L'analyse démontre qu'une bulle de voyage sous-régionale CAREC réduirait l'écart de touristes pour plusieurs de ses membres. Les bulles de voyages sous-régionales ne sont cependant une meilleure solution aux bulles bilatérales que lorsqu'il y a un grand nombre de voyages 
intra-sous-régionaux. Les considérations épidémiologiques peuvent également devenir encore plus complexes. (Matthias Helble et Anna Fink, 2020 :12) (Reprise du tourisme durant la pandémie de coronavirus)

L'une des résultats les plus importantes des bulles sous-régionales est l'établissement de protocoles harmonisés pour les voyages et le tourisme. Lesdits protocoles doivent prendre en compte l'itinéraire complet du client, de son taxi à l'aéroport jusqu'à son arrivée à l'hébergement et les visites des attractions et des sites. La Banque asiatique de développement (ADB) travaille actuellement avec des organisations internationales de voyage et de tourisme pour contribuer à ce processus. (Matthias Helble et Anna Fink, 2020 :12) (Reprise du tourisme durant la pandémie de coronavirus)

\section{Conclusion}

L'Organisation mondiale de la santé, en tant qu'un organisme international peut jouer un rôle primordial à côtés des Médecins Sans Frontières, des médecins du monde et des organisations non-gouvernementales internationales. Cet article a traité la pandémie du coronavirus et ses impacts sur divers pays de la région et sur le plan international. Il y a une vision selon laquelle les gouvernements ne sont plus en mesure d'intervenir dans certaines questions en vue de la mondialisation à savoir les secteurs de la sécurité et de la santé et de les diriger; A cet égard, les gouvernements voient leur rôle minoré et ils doivent conférer une partie de leurs fonctions aux institutions régionales transnationales mais dans la situation actuelle et par la pandémie du coronavirus, cette vision est exclue. II est évident que dans telles situations, en raison de l'ordre régional et international anarchique, les États de la région se contentent à leurs politiques nationales et doivent prêter attention au principe de l'auto-assistance et préférer les intérêts nationaux aux intérêts internationaux. Ainsi, les impacts de la pandémie de coronavirus sur les pays de la région de l'Asie occidentale, tels que la pauvreté, la récession, l'affaiblissement de la sécurité sanitaire, l'insatisfaction des groupes sociaux, etc., amènent l'avenir de l'ordre régional de l'Asie occidentale à s'orienter en termes de gestion, de plus en plus vers les États en tant qu'acteurs dominants, vers les rivalités et les divergences des modèles de communication et vers le principe d'autoassistance au lieu de la stratégie du multilatéralisme et à ne pas se joindre au processus de la mondialisation. Vu les effets négatifs laissés sur des pays de cette région, surtout les pays fragiles, on peut affirmer que l'ordre régional verra dans un proche avenir, une fragilisation encore plus forte des gouvernements fragiles, l'expansion du terrorisme, le renforcement des rivalités géopolitiques et l'intervention des puissa nces étrangères. Et c'est une image de l'avenir éventuel de l'ordre régional d'Asie occidentale qui va à l'encontre de celle brossée par ceux qui portent un regard optimiste à l'ordre régional post-Covid. En conséquence, l'ordre régional post-covid sera de plus en plus basé sur les politiques économiques et sécuritaires nationales, et la concurrence et les tensions ne donneront pas pour autant l'occasion de convergence et d'expansion de la coopération multilatérale.

\section{Bibliographie}

-Comprehensive Response to COVID-19: Saving Lives, Protecting Societies, Recovering Better, 
United Nations, JUNE 2020;

-Coronavirus disease 2019 (COVID-19) Addressing the impacts of COVID-19 in food crises, FAO, 2020

-GAUB Florence, BOSWINKEL Lotje, The geopolitical implications of the COVID-19 pandemic, European Parliament, 2020;

- HAN Emeline, et al., Lessons learnt from easing COVID-19 restrictions: an analysis of countries and regions in Asia Pacific and Europe, Health Policy, 2020, Vol 396, 1525

-HELBLE Matthias, FINK Anna, Reviving Tourism amid the COVID-19 Pandemic, ADB BRIEFS, 2020, NO. 150

-LORENTE Luis Miguel Lázaro, ARRABAL Ana Ancheta and PULIDO-MONTES Cristina, The Right to Education and ICT during COVID-19: An International Perspective, Sustainability 2020, 12, 9091;

-MONSHIPOURI, M., E. Neil et al. (2008) Constructing Human Rights in the Age of Globalization, traduit par Mehdi Zakerian,Tehran: Institute of Cultural \& Social Studies, 502-507.

-MONSHIPOURI, M. and M. Zakerian (2016) 'The State of Human Rights in Iran', cité par M. Mahmoud (ed.), Inside the Islamic Republic Social Change in Post-Khomeini Iran, Oxford University Press, 154-155.

-Y. AN Brian, TANG Shui-Yan, Lessons From COVID-19 Responses in East Asia: Institutional Infrastructure and Enduring Policy Instruments, American Review of Public Administration, 2020, Vol. 50(6-7);

-ZAKERIAN, M. (2014) Toward a Conceptual framework for the Internationally Human Rights, Mizan Publication. (third edition).

-ZAKERIAN, M. (2021) All Human Rights for All, Mizan Publication, (2nd edition) 61-65.

-ZAKERIAN, M. (2017) Policymaking of Human Rights in Middle East, Khorsandi Publication, 99-114. 